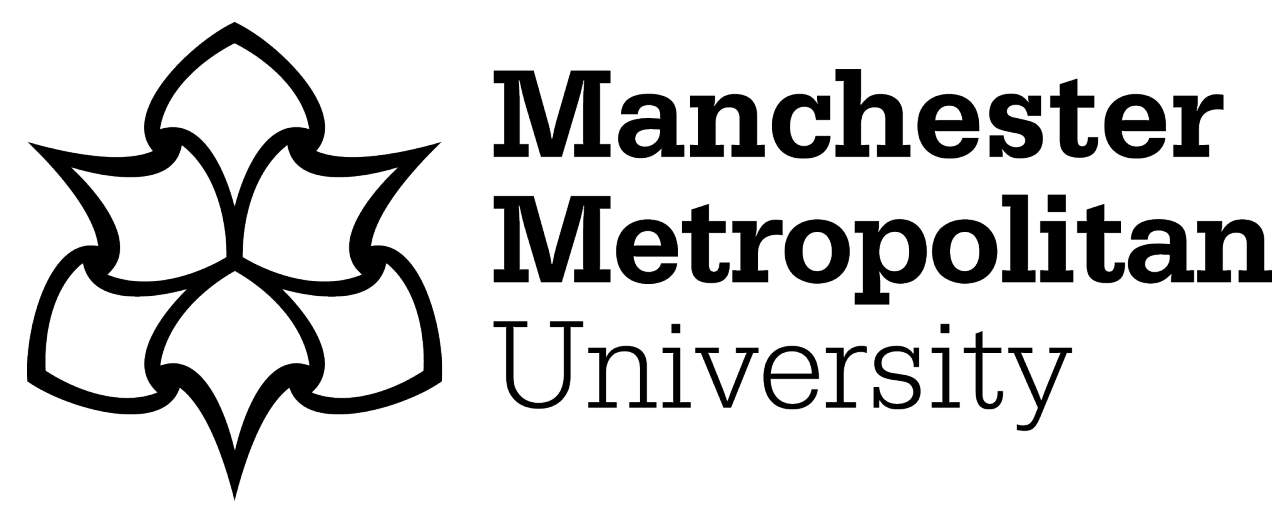

Crapnell, Robert D, Bernalte, Elena, Ferrari, Alejandro Garcia-Miranda, Whittingham, Matthew J, Williams, Rhys J, Hurst, Nicholas J and Banks, Craig E (2022) All-in-One Single-Print Additively Manufactured Electroanalytical Sensing Platforms. ACS Measurement Science Au, 2 (2). pp. 167-176. ISSN 2694-250X

Downloaded from: https://e-space.mmu.ac.uk/629054/

Version: Published Version

Publisher: American Chemical Society (ACS)

DOI: https://doi.org/10.1021/acsmeasuresciau.1c00046

Usage rights: Creative Commons: Attribution-Noncommercial-No Derivative Works 4.0

Please cite the published version 


\section{All-in-One Single-Print Additively Manufactured Electroanalytical Sensing Platforms}

Robert D. Crapnell, Elena Bernalte, Alejandro Garcia-Miranda Ferrari, Matthew J. Whittingham, Rhys J. Williams, Nicholas J. Hurst, and Craig E Banks*

Cite This: https://doi.org/10.1021/acsmeasuresciau.1c00046

Read Online

ACCESS |

Wl Metrics \& More

回 Article Recommendations

S1 Supporting Information

ABSTRACT: This manuscript provides the first report of a fully additively manufactured (AM) electrochemical cell printed all-in-one, where all the electrodes and cell are printed as one, requiring no postassembly or external electrodes. The three-electrode cell is printed using a standard non-conductive poly(lactic acid) (PLA)-based filament for the body and commercially available conductive carbon black/PLA (CB/ PLA, ProtoPasta) for the three electrodes (working, counter, and reference; $\mathrm{WE}, \mathrm{CE}$, and $\mathrm{RE}$, respectively). The electrochemical performance of the cell is evaluated first against the well-known nearideal outer-sphere redox probe hexaamineruthenium(III) chloride (RuHex), showing that the cell performs well using an AM electrode as the pseudo-RE. Electrochemical activation of the WE via chronoamperometry and $\mathrm{NaOH}$ provides enhanced electrochemical

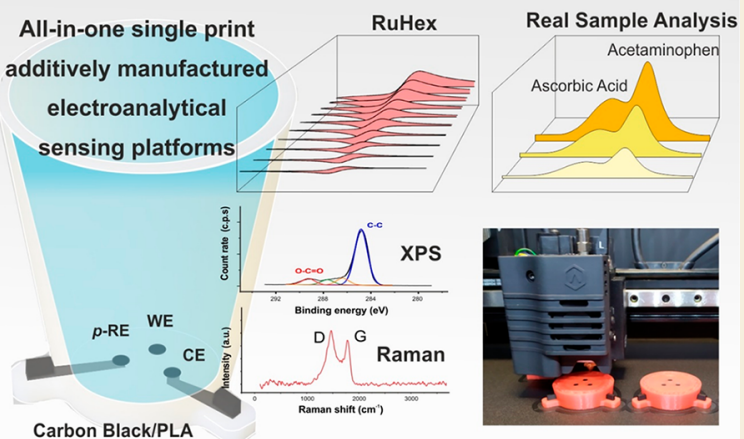
performances toward outer-sphere probes and for electroanalytical performance. It is shown that this activation can be completed using either an external commercial $\mathrm{Ag} / \mathrm{AgCl} \mathrm{RE}$ or through simply using the internal AM CB/PLA pseudo-RE and CE. This all-inone electrochemical cell (AIOEC) was applied toward the well-known detection of ascorbic acid (AA) and acetaminophen (ACOP), achieving linear trends with limits of detection (LODs) of $13.6 \pm 1.9$ and $4.5 \pm 0.9 \mu \mathrm{M}$, respectively. The determination of AA and ACOP in real samples from over-the-counter effervescent tablets was explored, and when analyzed individually, recoveries of 102.9 and $100.6 \%$ were achieved against UV-vis standards, respectively. Simultaneous detection of both targets was also achieved through detection in the same sample exhibiting 149.75 and $81.35 \%$ recoveries for AA and ACOP, respectively. These values differing from the originals are likely due to electrode fouling due to the AA oxidation being a surface-controlled process. The cell design produced herein is easily tunable toward different sample volumes or container shapes for various applications among aqueous electroanalytical sensing; however, it is a simple example of the capabilities of this manufacturing method. This work illustrates the next step in research synergising $\mathrm{AM}$ and electrochemistry, producing operational electrochemical sensing platforms in a single print, with no assembly and no requirements for exterior or commercial electrodes. Due to the flexibility, low-waste, and rapid prototyping of AM, there is scope for this work to be able to span and impact a plethora of research areas.

KEYWORDS: Additive Manufacturing, Electrochemistry, Electrodes, 3D-Printing, Electrochemical Cell, Ascorbic Acid (AA),

Acetaminophen (ACOP), all-in-one

\section{INTRODUCTION}

Research into additive manufacturing (AM) has become increasingly popular due to the ability to create complex designs, customizability, on-site manufacturing, and reduced costs for short production runs. It has transcended many industrial and academic categories, with various research groups across the globe pushing the boundaries of $3 \mathrm{D}$ and 4D manufactured items. ${ }^{1}$ There are many examples of research groups utilizing AM to overcome unique challenges in their work, producing bespoke designs for specific applications such as in situ Raman cells, ${ }^{2,3}$ thermal sensing of proteins ${ }^{4,5}$ or bacteria, ${ }^{4}$ and flow cells for monitoring the hydrogen evolution reaction. ${ }^{6} \mathrm{AM}$ has been used in a variety of ways for electrochemical research, from the production of electrodes to the development of bespoke cells and accessories for individual research needs. ${ }^{7}$ For the production of electrodes, recent work has focused on the use of fused filament fabrication (FFF) due to its continuing reduction in cost of entry, rapid prototyping capabilities, and extremely lowwastage. $^{8}$ In this technique, a continuous filament of thermoplastic material is fed through an extruder at elevated

Received: November 8, 2021

Accepted: November 30, 2021 
temperatures (above the melting point of the polymer) and deposited through a nozzle into pre-designed shapes in a layerby-layer process. ${ }^{9}$ The electrodes for these systems are typically made from conductive filaments, where conductive substances, typically carbon-based such as carbon black or graphene, are embedded into the thermoplastic filament matrix. Throughout the literature, researchers tend to use the commercially available conductive filaments; however, there are examples of groups producing their own bespoke filaments to high specifications for applications such as batteries, ${ }^{10}$ water splitting, ${ }^{11}$ and electrochemical sensing. ${ }^{12}$ In terms of the electrochemical sensing, research has focused predominantly on finding better ways to activate electrodes post-printing to benefit their specific applications. The activation of electrodes typically revolves around the removal of excess plastic to reveal increased amounts of the conductive materials. This can be accomplished in various ways such as electrochemical cycling in $\mathrm{NaOH},{ }^{8}$ reagentless laser treatment, ${ }^{13}$ or reducing agents. ${ }^{14}$ There have been examples reported in the literature of fully additively manufactured electrochemical platforms for reactions, ${ }^{15,16}$ water splitting, ${ }^{17,18}$ drug detection, ${ }^{19}$ and monitoring cellular and molecular events ${ }^{20}$ to name a few. These examples utilize various different methods of AM, such as selective laser melting (SLM), stereolithography (SLA), and FFF, often combining many of these. However, all of these platforms involve separate prints of different materials, meaning postprint processing is required along with assembly of the system. Lee et al. ${ }^{18}$ reported an AM electrochemical water splitting cell that utilized SLM for the production of $\mathrm{Ti}$ and Ni electrodes, PolyJet printing for the anodic and cathodic chambers, and FFF to produce a low-cost plastic locating base. Richter et al. ${ }^{21}$ reported a fully AM cell for the detection of dopamine that utilized FFF and a commercial carbon black/poly(lactic acid) (CB/PLA) filament for all three electrodes; however, they partially physically coated the reference electrode with silver ink to provide a similar reference to the commercial $\mathrm{Ag} \mid \mathrm{AgCl}$. Additionally, many of these systems that require holding solutions do so through the use of commercial o-rings to help seal the cells. This post-processing and modification of AM electrochemical platforms increases complexity, production time, and cost of each sensor. In this manuscript, we present the first all-in-one full PLA-based electrochemical platform (AIOEC) capable of being produced in a single print with no post-print assembly. This is a significant step forward for the synergistic use of FFF with electrochemistry and for the development of rapid, low-waste sensing platforms. This work highlights the versatility of the FFF process and indicates how this technology could be used to span multiple research areas, producing novel, on-the-spot solutions to specific research problems.

\section{EXPERIMENTAL SECTION}

\section{Materials}

All chemicals used were of analytical grade and were used as received without any further purification. All solutions were prepared with deionized water of resistivity not less than $18.2 \mathrm{M} \Omega \mathrm{cm}$ from a MilliQ system (Merck, Gillingham, UK). Hexaamineruthenium(III) chloride (RuHex), ascorbic acid (AA), acetaminophen (ACOP), sodium hydroxide, and phosphate-buffered saline (PBS) tablets were purchased from Merck (Gillingham, UK). Potassium chloride was purchased from Fisher Scientific (Loughborough, UK). The nonconductive poly(lactic acid) (PLA) filament used was the Red Raise3D Premium PLA (1.75 mm, Raise3D, California, US). The conductive PLA filament used was a commercial carbon black/PLA filament (1.75 mm, Protopasta, Vancouver, Canada). Real samples (effervescent tablets) of Superdrug Vitamin C $1000 \mathrm{mg}$ (Superdrug, UK) and Pandadol ActiFast soluble tablets (500 mg, GlaxoSmithKline, Middlesex, UK) were purchased from a local convenience store.

\section{Additive Manufacturing Parameters}

The AM all-in-one cell was produced using FFF on a Raise3D E2 3Dprinter (Raise3D, California, US). All designs and STL files were produced using Autodesk Fusion 360 and then sliced and converted to G-code files using the open source software ideaMaker 4.0.1 (Raise3D, California, US). The STL files are available for download from the SI. The dimensions of the AIOEC (CB/PLA) are shown in Figure S1, including schematics of the electrodes and cell sizes (A), a photograph of the prototype printed object (B) made from different colored non-conductive PLA, and a photograph of the printing process $(\mathrm{C})$, taken just after the electrodes have finished printing. The cells were all printed using the Raise3D E2 independent dual extruder (IDEX) printer with non-conductive Red Raise3D Premium PLA printed on the left nozzle $(0.4 \mathrm{~mm})$ at a set temperature of $210{ }^{\circ} \mathrm{C}$ and conductive carbon black/PLA Protopasta printed on the right nozzle $(0.4 \mathrm{~mm})$ at a set temperature of $220^{\circ} \mathrm{C}$. The printing bed temperature was set at $50{ }^{\circ} \mathrm{C}$ throughout the prints. The all-in-one cells were printed using a layer height of $0.1 \mathrm{~mm}$, shell width of $1 \mathrm{~mm}$, gyroid infill of $20 \%$, and infill speed of $70 \mathrm{~mm} / \mathrm{s}$ for the standard PLA profile and $100 \%$ infill and $35 \mathrm{~mm} / \mathrm{s}$ speed for the CB/PLA. The cells were typically printed in batches of three to test the reproducibility of the print, and although no observable leaking was detected, there was a minority of prints with a loss of calibration over time when using these independent dual extruders; this can be fixed by calibrating the printer and reprinting the item. Figure S2 shows the progress of the printing, being a bottom-up print, showing how the height of the AIOEC increases until the end print. This print had a purge block located close to the cells as well as a skirt to help prime the nozzle prior to printing the first layer and between each extruder change. The print for three full $50 \mathrm{~mL}$ volume AIOECs took $16 \mathrm{~h} 40 \mathrm{~min}$ and $34 \mathrm{~s}$ to complete using a total of $71.9 \mathrm{~g}$ of standard PLA and $2.9 \mathrm{~g}$ of CB/ PLA, with an estimated material cost of $£ 2.25$ per three cells printed simultaneously. The all-in-one cells shown herein are just a mere example of what additive manufacturing can do in terms of simplicity and easiness; this setup allows the use of external magnetic stirrers, a tidy connection, and organized connection cables. The $50 \mathrm{~mL}$ volume allows its use for standard addition methodologies. If one wants to alter its volume, it can be easily achieved by modifying the CAD file, reslicing the design, and printing it. This is a major advantage of AM.

\section{Electrochemical Experiments}

A $\mu$-Autolab $128 \mathrm{~N}$ potentiostat (Utrecht, The Netherlands) was used in conjunction with NOVA 2.1.5 (Utrecht, The Netherlands) to carry out electrochemical measurements using a three-electrode configuration. Throughout the work, AM working electrodes were used with either an external nickel wire counter electrode (CE) and commercial $\mathrm{Ag} \mid \mathrm{AgCl}$ reference electrode (RE) or the internal printed $\mathrm{CB} / \mathrm{PLA} \mathrm{CE}$ and pseudo-RE. Scan rate studies against the ideal outer-sphere redox probe RuHex $(1 \mathrm{mM}$ in $0.1 \mathrm{M} \mathrm{KCl})$ were performed using cyclic voltammetry $\left(0.005-0.3 \mathrm{~V} \mathrm{~s}^{-1}\right)$ from +0.3 to $-0.6 \mathrm{~V}$ using the external reference and counter and from 0 to $-1.0 \mathrm{~V}$ using the pseudoreference and counter. All solutions of RuHex were degassed thoroughly for at least $15 \mathrm{~min}$ with nitrogen prior to any electrochemical measurement. The activation of the working electrodes was performed using chronoamperometry in a solution of $\mathrm{NaOH}(0.5 \mathrm{M})$ by holding the cell at a potential of $+1.4 \mathrm{~V}$ for 200 $\mathrm{s}$, followed by $-1.0 \mathrm{~V}$ for $200 \mathrm{~s}$ unless otherwise stated. ${ }^{21}$ The detection of all analytes using $\mathrm{CV}$ was performed at a scan rate of 50 $\mathrm{mV} \mathrm{s}^{-1}$, and a standard addition method was used for calibration $(n=$ 3). Differential pulse voltammetry (DPV, $10 \mathrm{mV} \mathrm{s}^{-1}$ ) was used for the detection in real samples from -0.1 to $+1.0 \mathrm{~V}$, with a step potential of $0.005 \mathrm{~V}$, modulation amplitude of $0.025 \mathrm{~V}$, modulation time of $0.05 \mathrm{~s}$, and interval time of $0.5 \mathrm{~s}$. 


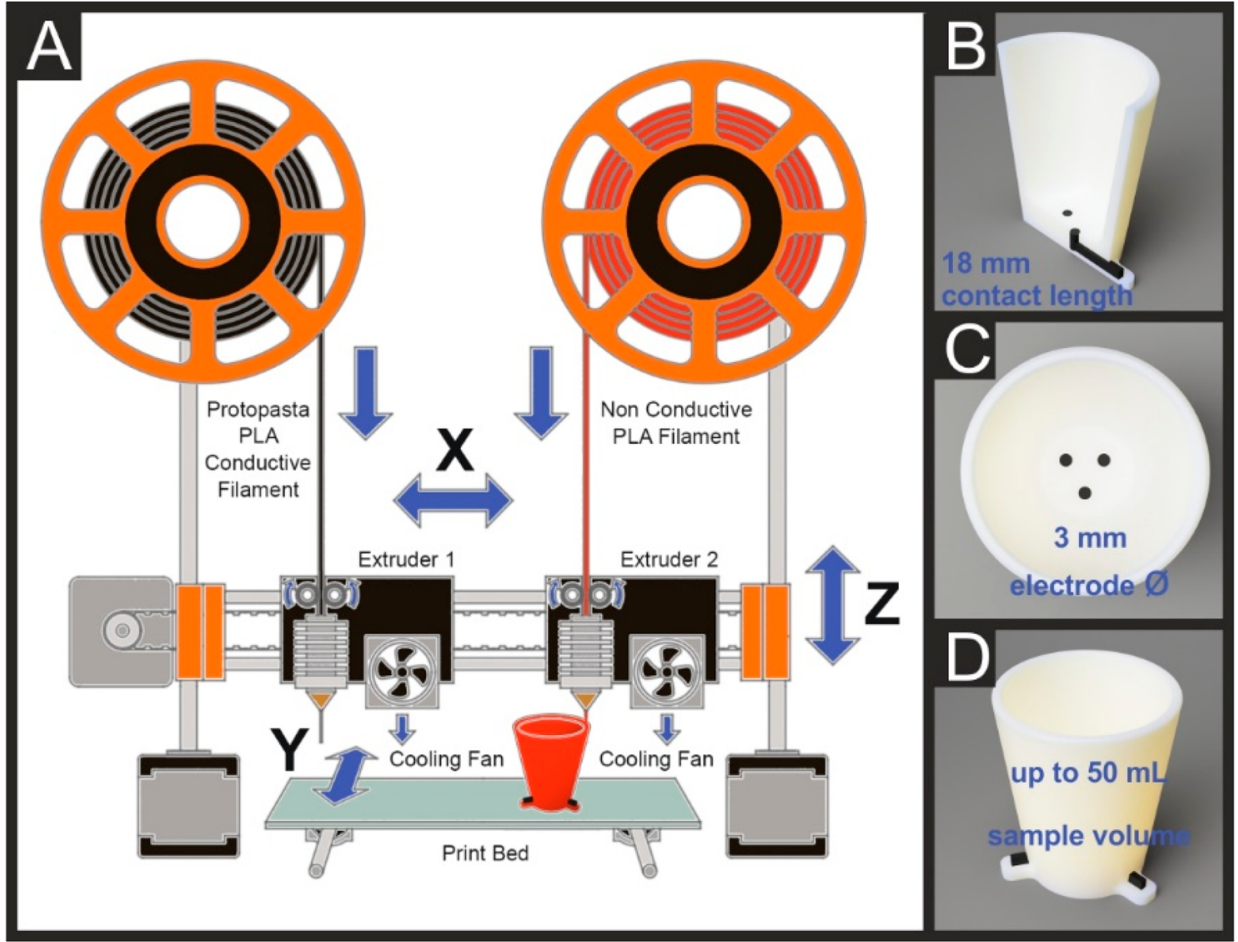

Figure 1. (A) Schematic of the FFF printing of the AM all-in-one cell highlighting the independent dual extruders used, one loaded with commercial conductive carbon black/PLA (Protopasta) and the other loaded with non-conductive PLA. (B) CAD cut view of the AM all-in-one cell, highlighting how the electrode is printed inside the non-conductive PLA with an electrode connection length of $18 \mathrm{~mm}$. (C) Top view of the AM all-in-one cell highlighting the three evenly spaced identical electrodes with a $3 \mathrm{~mm}$ diameter. (D) Side view of the AM all-in-one cell, showing the connections for the electrodes, designed to easily attach to standard crocodile clips. The cell used throughout this work is designed to house up to $50 \mathrm{~mL}$ of solution, making electrochemical and electroanalytical experiments simple.

\section{Surface Characterization}

Scanning electron microscopy (SEM) measurements were recorded on a Supra 40VP Field Emission (Carl Zeiss Ltd., Cambridge, UK) with an average chamber and gun vacuum of $1.3 \times 10^{-5}$ and $1 \times 10^{-9}$ mbar, respectively. Samples were mounted onto aluminum SEM pin stubs (12 mm diameter, Agar Scientific, Essex, UK). X-ray Photoelectron Spectroscopy (XPS) data was acquired using an AXIS Supra (Kratos, UK), equipped with an $\mathrm{Al}$ X-ray source (1486.6 $\mathrm{eV}$ ) operating at $300 \mathrm{~W}$ in order to perform survey scans and $450 \mathrm{~W}$ for narrow scans. All X-rays were monochromated using a $500 \mathrm{~mm}$ Rowland circle quartz crystal X-ray mirror. The angle between the Xray source and analyzer was $54.7^{\circ}$ with an electron energy analyzer: $165 \mathrm{~mm}$ mean radius hemispherical sector analyzer operating in fixed analyzer transmission mode, pass energy of $160 \mathrm{eV}$ for survey scans, and $40 \mathrm{eV}$ narrow scans. A detector with a delay line detector with multichannel plate was utilized.

Raman spectroscopy was performed using an XploRA PLUS (Horiba, UK) fitted with a $638 \mathrm{~nm}$ excitation laser at a power of 3 $\mathrm{mW}$ to avoid any heating effects. Spectra were recorded using a $10 \mathrm{~s}$ exposure time for 10 accumulations at each spectra, between the region of 250 to $3600 \mathrm{~cm}^{-1}$.

\section{Real Sample Analysis}

For real samples, the effervescent tablets were initially dissolved in $250 \mathrm{~mL}$ of deionized water. For the standard addition experiment, a 4:1 AA to ACOP dilution of real samples containing 100 and $25 \mu \mathrm{M}$, respectively, was prepared in $0.01 \mathrm{M}$ PBS ( $\mathrm{pH}$ 7.4). For the electrochemical analysis, $10 \mathrm{~mL}$ of such solution was added to the $\mathrm{AM}$ cell, and standard additions were performed by adding $10 \mathrm{mM}$ stock solutions of AA and ACOP standards freshly prepared in $25 \mathrm{~mL}$ of 0.01 M PBS ( $\mathrm{pH}$ 7.4). Note that the standards were prepared separately and added individually for the calibration. All UV-vis measurements were performed using a Thermo Scientific Evolution 201 UV-visible Spectrophotometer with a quartz cuvette of path length $1 \mathrm{~cm}$. A blank sample of PBS $(0.01 \mathrm{M})$ was used as the background measurement. Absorbance at 266 or $243 \mathrm{~nm}$ was taken as the peak absorbance for ascorbic acid and acetaminophen, respectively. A range of concentrations of ascorbic acid (10.09, $20.59,51.14$, and $104.27 \mu \mathrm{M})$ and acetaminophen $(11.34,62.15$, 92.84, and $123.12 \mu \mathrm{M}$ ) were produced in PBS for the calibration of the system. For real samples, the solutions prepared above were tested without further modification to measure the peak absorbance. This was used in conjunction with the calculated absorption coefficient to calculate the initial concentration in the tablets.

\section{RESULTS AND DISCUSSION}

In this manuscript, we present, for the first time, an all-in-one single-print electrochemical cell (AIOEC) including working, counter, and reference Protopasta (carbon black (CB)//PLA) electrodes. The use of an independent dual extruder FFF printer allows the construction of a water-tight, operational cell design straight from the print bed comprised of two different materials, one non-conductive and one electrically conductive.

\section{Electrochemical Cell Design and Printing}

The electrochemical cell was designed to mimic a simple inverted conical vessel, one of the simplest experimental setups used throughout electrochemical laboratories, however, with the electrodes already printed into the structure. To achieve this, an independent dual extruder 3D-printer was utilized, where non-conductive PLA was printed from one extruder, and the conductive PLA was printed using the other extruder, Figure 1A. The cell was designed to have all of the electrodes identical in size, shape, and spacing to allow for any electrode to be attached to any of the cables on the potentiostat and have the system perform identically. The cell volume is $50 \mathrm{~mL}$ to 


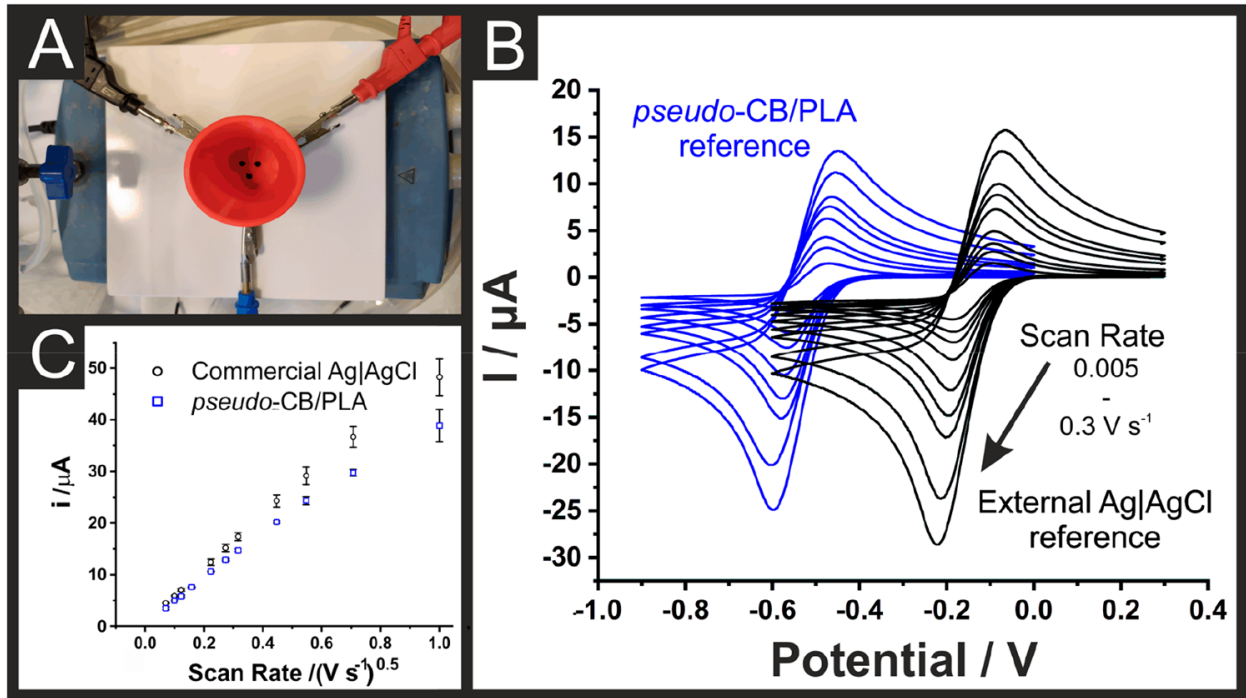

Figure 2. (A) Overhead photograph of the electrochemical setup for the AM all-in-one cell, with the working, counter, and reference cables attached to the electrodes through crocodile clips. (B) Cyclic voltammograms corresponding to the scan rate study $\left(0.005-0.3 \mathrm{~V} \mathrm{~s} \mathrm{~s}^{-1}\right)$ of hexaamineruthenium(III) chloride $(1 \mathrm{mM})$ in $\mathrm{KCl}(0.1 \mathrm{M})$, performed using an AM carbon black/PLA working electrode (3 mm) in conjunction with either an AM carbon black/PLA counter $(3 \mathrm{~mm}$ ) and AM carbon black/PLA pseudo-reference ( $3 \mathrm{~mm})$ (blue lines) or an external nickel wire counter and external commercial $\mathrm{Ag} \mid \mathrm{AgCl}$ reference (black lines). (C) Plot of the average peak current versus the square root of the scan rate for the AM all-in-one cell using the internal AM carbon black/PLA pseudo-reference (3 mm) (blue) and the external commercial AglAgCl reference (black).

Table 1. Peak-to-Peak $\left(\Delta E_{\mathrm{P}}\right)$ Separation, Heterogeneous Electron Transfer Rate Constants $\left(k_{\text {eff }}^{0}\right)$, and Electrochemical Active Area $\left(A_{\text {real }}\right)$ Values Determined Using $1 \mathrm{mM}$ RuHex in $0.1 \mathrm{M} \mathrm{KCl}$

$\begin{array}{lccc} & \Delta E_{\mathrm{p}} / \mathrm{mV}\left(@ 50 \mathrm{mV} \mathrm{s}^{-1}\right) & k_{\text {eff }}^{0} / \mathrm{cm} \mathrm{s}^{-1} & A_{\text {real }} / \mathrm{cm}^{2} \\ \text { AM CB/PLA with pseudo-RE } & 97.66 & 2.48 \times 10^{-3} \pm 3.13 \times 10^{-5} & 0.0581 \\ \text { AM CB/PLA with external RE AglAgCl } & 103.35 & 2.71 \times 10^{-3} \pm 1.65 \times 10^{-4} & 0.0886\end{array}$

allow its use for standard addition methodologies or benchmarking against external counter or reference electrodes. The flexibility of scaling up-/down- designs is one of the biggest advantages of AM, allowing simple modifications of the computer file to translate on to the final print design. The electrode areas are defined (and insulated) by the nonconductive PLA printed around them and connected to the potentiostat through an $18 \mathrm{~mm}$ printed stem, Figure $1 \mathrm{~B}$, that goes beneath the electrode and then horizontally through the vessel. The electrodes consist of $3 \mathrm{~mm}$ diameter discs printed from the conductive carbon black/PLA commercial filament Protopasta, evenly spaced around a $10 \mathrm{~mm}$ diameter circle in the bottom of the vessel, Figure 1C. The stems of the electrodes protrude from the vessel walls allowing for a simple connection to the potentiostat through the attachment of standard crocodile clips, Figure 1D, while a non-conductive bottom platform prevents shorting of the clips with work surfaces/apparatus. This design was printed in triplicate throughout this work, Figure S1C, emphasizing the reproducibility of the print. This triplicate print was designed with a single-layer nozzle priming skirt and a wipe/purge block located near the prints to help prime the nozzle prior to printing each layer, ensuring a high surface quality, which was vital to achieve a water-tight print. This three-cell print took a total print time of $16 \mathrm{~h} 40 \mathrm{~min}$ and $34 \mathrm{~s}$ to complete, using a total of $71.9 \mathrm{~g}$ of non-conductive filament and $2.9 \mathrm{~g}$ of conductive filament, coming out at an estimated material cost of $£ 2.25$ per print (for three cells). We note that the time and cost could be reduced significantly by printing vessels for a smaller working volume, as this design was capable of working up to volumes of $50 \mathrm{~mL}$. Once a suitable design and reproducible water-tight print were achieved, the electrochemical performance of the cell was characterized.

\section{Electrochemistry at the Single-Printed AM Electrochemical Setup}

In order to electrochemically characterize the electron transfer kinetic properties of our AIOEC, the near-ideal outer-sphere hexaammineruthenium(III) chloride (RuHex) probe was explored. Scan rate studies with $\mathrm{RuHex}$ are a common benchmarking tool due to the RuHex's dependence only on the electronic structure (DoS) of carbon-based electrode materials and will therefore give useful insights into the electrochemically active sites of the printed surfaces. An overhead view of the electrochemical setup for this system is presented in Figure 2A, where each printed electrode is simply attached through a crocodile clip. The electrochemical performance of the cell was tested utilizing both a standard commercial $\mathrm{Ag} \mid \mathrm{AgCl}$ reference electrode alongside a nickel wire counter electrode and using the built-in printed carbon black/PLA pseudo-reference electrode and printed counter electrode. The cyclic voltammetric scan rate studies corresponding to the reduction and oxidation of RuHex ( $1 \mathrm{mM}, 0.1$ $\mathrm{M} \mathrm{KCl}$ ) are presented in Figure 2B, showing that a response is possible using both setups, although the pseudo-reference exhibits an expected potential shift to more negative potentials. This data can be utilized to obtain vital information about the performance of the electrochemical cell. Table 1 includes the peak-to-peak separation $\left(\Delta E_{\mathrm{P}}\right)$, heterogeneous electron transfer 


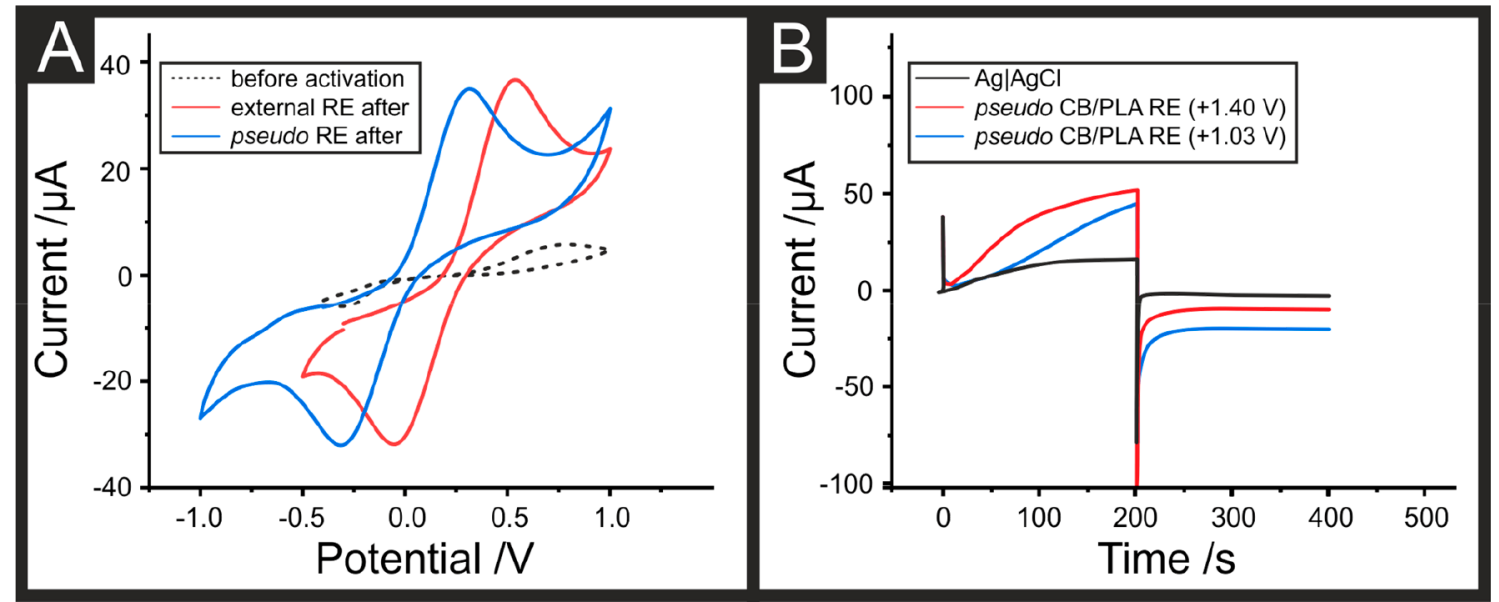

Figure 3. Electrochemical characterization of the activation of the AIOEC working electrodes. (A) Ferro-/ferricyanide ( $1 \mathrm{mM}$; in $0.1 \mathrm{M} \mathrm{KCl)}$ cyclic voltammograms obtained using untreated (before activation; black) and activated AM CB/PLA using the external reference electrode (RE) and AM CB/PLA pseudo-RE (red and blue), respectively. (B) Activation of the AM all-in-one cell working electrodes using a chronoamperometry activation plot of the AM CB/PLA working electrode (and electrochemical cell) corresponding to the application of $+1.4 \mathrm{~V}$ for $200 \mathrm{~s}$ followed by $-1.0 \mathrm{~V}$ for $200 \mathrm{~s}$ using an external commercial $\mathrm{Ag} / \mathrm{AgCl}$ reference electrode (black); the same parameters using the internal AM carbon black/PLA pseudo-reference $(3 \mathrm{~mm})$ (red); and the application of $+1.03 \mathrm{~V}$ for $200 \mathrm{~s}$ followed by $-1.37 \mathrm{~V}$ for $200 \mathrm{~s}$ using the internal AM carbon black/PLA pseudo-reference $(3 \mathrm{~mm})$ (blue).

(HET) rates $\left(k_{\text {eff }}^{0}\right)$, and electroactive area $\left(A_{\text {real }}\right)$ for both three-electrode cell configurations. These values can be calculated from the peak potentials and currents obtained from the scan rate study. Figure $2 \mathrm{~B}$ depicts the scan rate studies for RuHex comparing the use of the external and the pseudo-REs (black and blue, respectively), and Figure 2C shows the current vs. scan rate values for both systems. A 0.37 $\mathrm{V}$ difference in the peak position $\left(E_{\mathrm{p}}\right)$ can be seen when comparing the use of the external $\mathrm{Ag} \mid \mathrm{AgCl} \mathrm{RE}$ to the $\mathrm{AM} \mathrm{CB} /$ PLA pseudo-RE, when comparing the reduction peaks for RuHex at $50 \mathrm{mV} / \mathrm{s}$. Both systems show similar peak-to-peak separations, Figure S3A, with 88 and $81 \mathrm{mV}(N=3)$ differences observed at slow scan rates $(5-25 \mathrm{mV} / \mathrm{s})$ for the external and pseudo systems, respectively. Table 1 shows that the calculated HET rates $\left(k_{\text {eff }}^{0}\right)$, calculated through the Nicholson equation, ${ }^{22}$ are $2.48 \times 10^{-3} \pm 3.13 \times 10^{-5}$ and $2.71 \times 10^{-3} \pm 1.65 \times 10^{-4} \mathrm{~cm} \mathrm{~s}^{-1}$ for the electrodes when compared using external and the internal pseudo-REs, respectively (at RuHex). Figure S3B plots the $\Delta E_{\mathrm{P}}$ comparison for external and pseudo-RE, exhibiting a good correlation between the use of both electrodes. The electrochemical active areas were calculated using the quasi-reversible RandlesŠevćik equation and cyclic voltammetry $(\mathrm{CV}) .{ }^{23-26}$ The scan rates utilized for the CVs were from 0.05 to $0.3 \mathrm{~V} / \mathrm{s}$, and the final electroactive areas were determined to be $0.089 \pm 0.06$ $\mathrm{cm}^{2}$ for the WEs (using external $\mathrm{Ag} \mid \mathrm{AgCl}(N=10)$ ), compared to the theoretical area of $0.071 \mathrm{~cm}^{2}$ for a $3 \mathrm{~mm}$ radius circle. This apparent increase in area could be due to surface texturing caused in part by the nozzle producing small ridges or insufficient adherence between the two different filaments, allowing solution to fill any voids present between the nonconductive and conductive PLA. Next, attention was turned to enhance the electrochemical performance of the working electrode by a pre-activation method. Although unnecessary for the use of RuHex due to its near-ideal outer-sphere nature, for the best performance in electroanalytical sensing applications toward other molecules, such activation has been found to be vital. To further enhance the electrochemical properties of AM electrodes, several literature reports have paid special attention to the enhancement of the electrochemical properties of AM PLA-graphene electrodes by their post-printing activation. Electrode activation can be in the form of physical (polishing), electrochemical, or chemical treatments that modify the electrode's surface and lead to an increase in surface area, defects, and electron transfer rates. Kalinke et al. ${ }^{8}$ performed and reported a diligent comparison of the different activation methods for AM PLA-graphene electrodes, reporting that the combined use of DMF, $\mathrm{NaOH}$, and a chronoamperometric method promoted an increase in their electrochemical response toward dopamine sensing. Observing their reported improvement in the electrochemical signatures, we utilize their activation methodology to our AIOEC as shown in Figure 3. Mechanical activation methods (such as polishing) would be difficult to apply in a sealed narrow vessel such as ours, and chemical activation methods would dissolve/damage the non-conductive PLA parts of the vessel. Consequently, electrochemical activation was chosen as the ideal method due to its ability to only modify those parts of the electrodes that are in contact with the electrolyte, namely the surface of the electrodes themselves. The first electrochemical study was using the well-known inner-sphere redox probe ferro-/ferricyanide $(1 \mathrm{mM}$ in $0.1 \mathrm{M} \mathrm{KCl})$. Figure $3 \mathrm{~A}$ shows the typical voltammograms for untreated (before activation; in black) and activated AM CB/PLA WEs using the external RE and pseudo-AM CB/PLA RE (red and blue), respectively. Upon inspection of Figure 3A, it can be seen that the activation method increases the electrochemical signature of the ferro-/ferricouple when compared to the untreated WE. It can also be seen that there is a difference in the peak position $\left(E_{\mathrm{p}}\right)$ when comparing the use of the external $\mathrm{Ag} \mid \mathrm{AgCl} \mathrm{RE}$ and the AM CB/PLA pseudo-RE; this potential shift is $+0.22 \mathrm{~V}$ at the oxidation peak when comparing the external to the pseudo$\mathrm{RE}$, which is similar to the one observed in Figures 2 and S3, following a similar trend. It can also be seen in Figure 3B that both voltammograms for the activated AM CB/PLA WE display comparable reversibility regimes, exhibiting $\Delta E_{\mathrm{P}}$ separations of 593 and $618 \mathrm{mV}$ for the ferro-/ferricouple when using the external and the pseudo-RE, respectively. The 


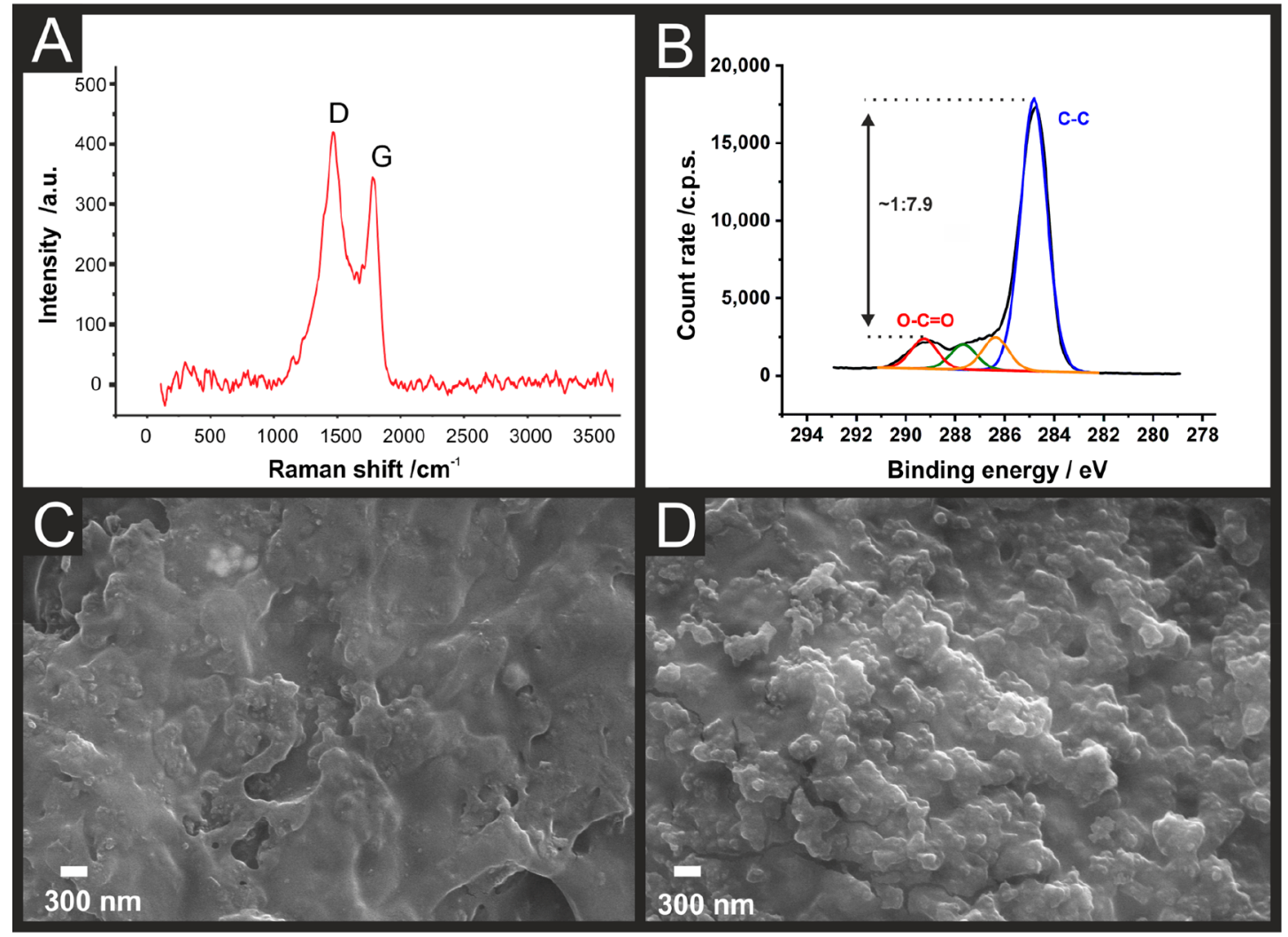

Figure 4. (A) Raman profile of an unused AM carbon black/PLA working electrode showing graphite's D and G bands at 1479 and $1791 \mathrm{~cm}^{-1}$, respectively. (B) XPS C 1s model for the surface of the AM carbon black/PLA working electrode activated using the internal AM carbon black/ PLA pseudo-reference $(3 \mathrm{~mm})$. (C) SEM image of the surface on an untreated AM carbon black/PLA working electrode. (D) SEM image of the surface of the AM carbon black/PLA working electrode activated using the internal AM carbon black/PLA pseudo-reference (3 mm).

electrochemical activation methodology involves a chronoamperometric method through the application of $+1.4 \mathrm{~V}$ for $200 \mathrm{~s}$ followed by $-1.0 \mathrm{~V}$ for $200 \mathrm{~s}$ using an external commercial Agl $\mathrm{AgCl} \mathrm{RE}$. For ease of user application, activation of this electrochemical platform through use of the in-built pseudoreference electrode would be ideal, removing any need for the use of more expensive commercial reference electrodes. This chronoamperometric method, compared to the one applied with the built-in pseudo-RE and the same method converted to the equivalent voltage to that of the pseudo-RE, are shown in Figure $3 \mathrm{~B}$ in red, blue, and black colors, respectively. The method adapted and applied to the pseudo-RE voltage equals to +1.03 and $-1.37 \mathrm{~V}$ for $200 \mathrm{~s}$ in both cases. As it can be seen, the use of an external $\mathrm{Ag} \mid \mathrm{AgCl} \mathrm{RE}$ exhibits the most positive current response when a voltage of $+1.4 \mathrm{~V}$ for $200 \mathrm{~s}$ was applied. Following that, the application of +1.40 and $+1.03 \mathrm{~V}$ when using the pseudo-CB/PLA RE exhibited less positive current responses. It is important to note that similar current values are being passed at the end of the chronoamperometric methods, showing that the activation method is working and is stable to some extent. This indicates that although the activation process could be slightly better and quicker using the external commercial reference electrode, it is possible using the pseudo-reference. This would allow any user to utilize this system and increase the ability for this platform to be used away from a laboratory environment. A thorough physicochemical characterization of the AM CB/PLA electrodes was then performed in order to determine their composition. This involved Raman spectroscopy, X-ray photoelectron spectros- copy (XPS), and scanning electron microscopy (SEM). Figure 4 shows the Raman, XPS, and SEM images from the AM CB/ PLA samples. The Raman spectra of the AM CB/PLA electrodes are shown in Figure 4A, displaying the welldocumented D and G Raman bands for graphite at 1479 and $1791 \mathrm{~cm}^{-1}$, respectively. The $\mathrm{G}$ band is related to the firstorder Raman band of all $\mathrm{sp}^{2}$ hybridized carbons. The $\mathrm{D}$ band is usually associated with defects and disorders due to the breathing modes of $\mathrm{sp}^{2}$ atoms in rings within graphite atoms and in rings within graphite layers. ${ }^{27-29}$ Next, XPS characterization was performed, and it can be seen upon inspection of the respective spectra that the ratio of $\mathrm{O}-\mathrm{C}=\mathrm{O}$ to $\mathrm{C}-\mathrm{C}$ groups is $1: 2$ for the untreated electrodes (Figure $\mathrm{S} 4 \mathrm{~A}$ ), being 1:8.2 (Figure S4B) and 1:7.9 (Figure 4B) for the activation of the electrodes when using the external and pseudo-reference electrodes, respectively (Figure 4B). This can be explained as the $\mathrm{O}-\mathrm{C}=\mathrm{O}$ bonding is only present in the PLA backbone of the electrodes, not the carbon black active material. When printed, the surface concentration of PLA is high, whereas after activation, PLA is stripped from the surface, presenting a higher concentration of the carbon black active material, hence the increase in observed $\mathrm{C}-\mathrm{C}$ bonding. The electrochemical responses described above (Figure 3) can also be correlated with the XPS results, where the activated electrodes exhibit faster and higher in current intensity electrochemical signatures toward the ferro-/ferricouple when compared to the nonactivated one. The chemical activation method via $\mathrm{NaOH}$ and the chronoamperometric method promote PLA saponification, exposing the active material's active sites and electrochemical 


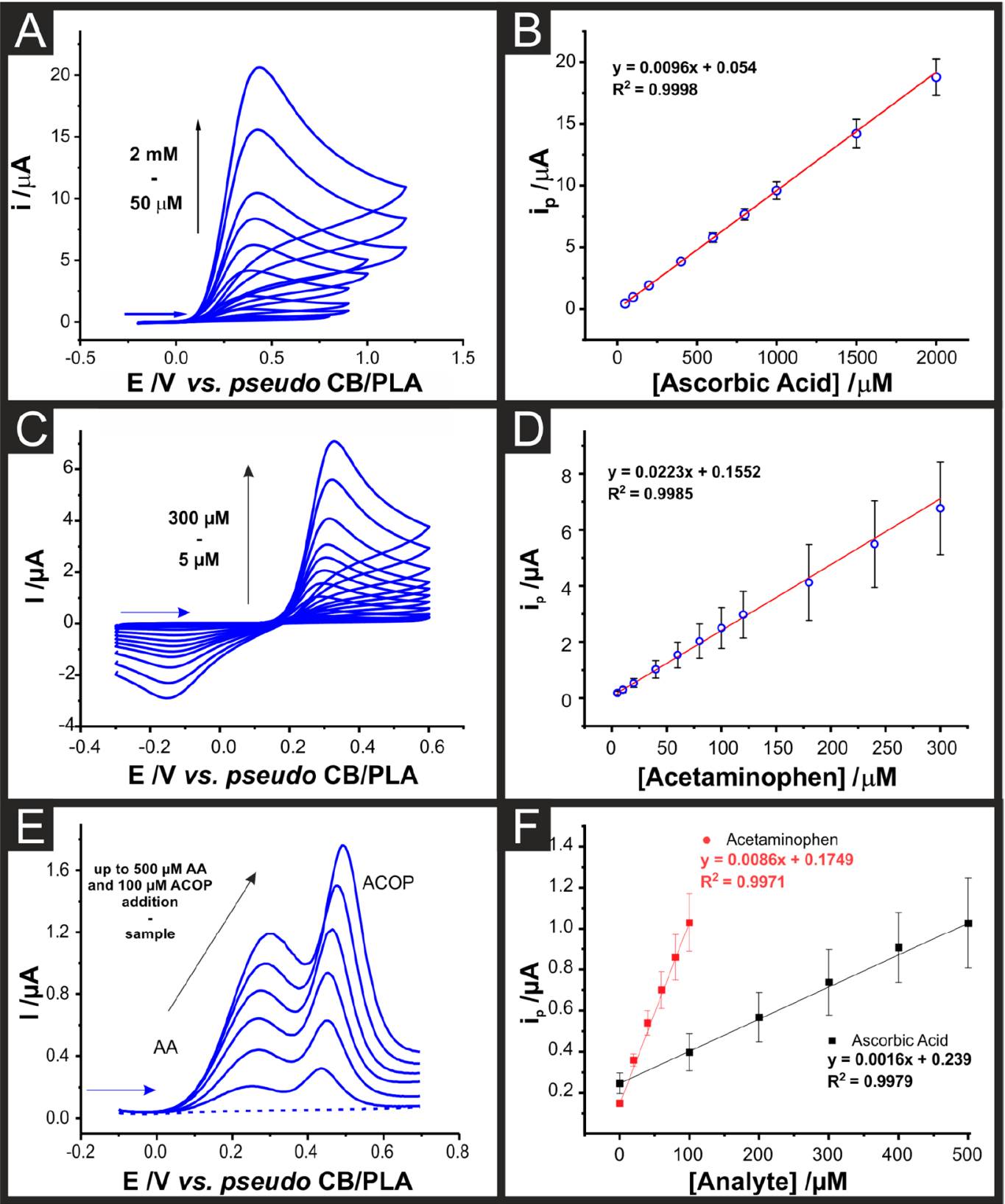

Figure 5. (A) Cyclic voltammograms $\left(0.05 \mathrm{~V} \mathrm{~s}^{-1}\right)$ for the detection of ascorbic acid $(0.05-2 \mathrm{mM})$ in $\mathrm{PBS}(0.01 \mathrm{M}, \mathrm{pH}=7.4)$. (B) Calibration curve corresponding to the cyclic voltammetric detection of ascorbic acid $(0.05-2 \mathrm{mM}, N=3)$ in $\mathrm{PBS}(0.01 \mathrm{M}, \mathrm{pH}=7.4)$. (C) Cyclic voltammograms $\left(0.05 \mathrm{~V} \mathrm{~s}^{-1}\right)$ for the detection of acetaminophen $(5-300 \mu \mathrm{M})$ in PBS $(0.01 \mathrm{M}, \mathrm{pH}=7.4)$. (D) Calibration curve corresponding to the cyclic voltammetric detection of acetaminophen $(5-300 \mu \mathrm{M}, N=3)$ in PBS (0.01 $\mathrm{M}, \mathrm{pH}=7.4)$. (E) Differential pulse voltammograms (DPVs) for the simultaneous detection of ascorbic acid and acetaminophen from commercial over-the-counter tablets with standard additions up to $500 \mu \mathrm{M}$ of ascorbic acid and $100 \mu \mathrm{M}$ of acetaminophen in PBS $(0.01 \mathrm{M}, \mathrm{pH}=7.4)$. (F) Calibration curve corresponding to the DPV detection of ascorbic acid and acetaminophen in PBS $(0.01 \mathrm{M}, \mathrm{pH}=7.4, N=3)$. All of the measurements were performed using the AIOEC with all three identical electrodes printed from carbon black/PLA ( $3 \mathrm{~mm}$ diameter).

active area and increasing the $\mathrm{C} / \mathrm{O}$ content, for which innersphere probes, such as a ferro-/ferricouple are beneficially influenced, resulting in the enhanced electrochemical responses and faster HET kinetics as seen in Figure 3. This is supported by the XPS ratios, where the ratio of $\mathrm{C}-\mathrm{C}$ to $\mathrm{O}-$ $\mathrm{C}=\mathrm{O}$ is higher at working electrodes that were activated using the external RE than those used with the built-in pseudo-RE. Figure 4C,D depicts the SEM images of the surface on an untreated and activated AM carbon black/PLA WE, showing an evident change in the surface roughness and exposure of edge plane sites available upon the electrode surface after the PLA saponification increased active sites.

\section{Electroanalytical Performance of the Single-Printed AM Cell}

We now turn to exploring the electroanalytical performance of these AM electrodes toward the electrochemical detection of well-known analytes ascorbic acid (AA) and acetaminophen (ACOP) in PBS (0.01 M, pH = 7.4). This was achieved through simple standard addition of the analytes to the electrochemical cell. Cyclic voltammetry was used to monitor 
the increasing magnitude of the oxidation peaks of the two analytes. The electrochemical oxidation of AA and ACOP is shown in Figure S5 (A and B, respectively). Figure 5 depicts the cyclic voltammograms of increasing amounts of AA (A), the calibration plot of AA (B), the cyclic voltammograms of increasing amounts of ACOP (C), and their respective calibration plot (D). The electrochemical sensitivities calculated for the AA and ACOP are 0.0096 and $0.0268 \mu \mathrm{M} / \mu \mathrm{A}$, with calculated limits of detection (LODs) of $13.6 \pm 1.9$ and $4.5 \pm 0.9 \mu \mathrm{M}$, respectively, for AA and ACOP. The cell was then tested toward the determination of AA and ACOP in real samples obtained from commercial over-the-counter (without a prescription) effervescent tablets from a local shop. The samples were prepared as explained in the Experimental Section, and the standard addition method was used to determine the concentration in the original tablets. The values obtained for the AA and ACOP are presented in Table 2, along

Table 2. Summary of the Mean Values of Ascorbic Acid and Acetaminophen Content Determined in Real over-theCounter Effervescent Tablets through Both UV-Vis and Using the AIOEC

\begin{tabular}{lcc} 
& \multicolumn{2}{c}{ sample } \\
\cline { 2 - 3 } technique & ascorbic acid $(\mathrm{mg})$ & acetaminophen $(\mathrm{mg})$ \\
UV-vis & $1050 \pm 18$ & $511 \pm 16$ \\
all-in-one cell & $1080 \pm 24$ & $514 \pm 7$
\end{tabular}

with the measured values using UV-vis as validation. The UV-vis data obtained for these real samples can be seen in Figure S6. As seen, both electrochemical measurements show an increased concentration of analyte compared to what was advertised on the packaging, $1000 \mathrm{mg}$ for AA and $500 \mathrm{mg}$ for ACOP. This was also the case seen when measuring the samples with UV-vis, leading to recoveries of 102.9 and $100.6 \%$ for AA and ACOP, respectively. Last, we address the simultaneous electrochemical determination of AA and ACOP in real samples. This is of interest as in some regions, tablets containing these two components are commonplace, although not in the UK. These solutions were prepared by mixing the two real samples measured above in a $4: 1$ ratio (AA:ACOP). Figure 5E shows the standard addition method used to quantify the amount of both AA and ACOP, by starting with the unknown undiluted sample, to which known additions of both of the analytes in fixed concentrations of $100 \mu \mathrm{M}$ of AA and $25 \mu \mathrm{M}$ of ACOP in PBS $(0.01 \mathrm{M}, \mathrm{pH}=7.4)$ were added to the electrochemical cell. DPV was used to simultaneously detect the analytes, as there was no resolution between the two peaks when using $\mathrm{CV}$. Figure $5 \mathrm{~F}$ shows the simultaneous calibration plots for both AA and ACOP done by the standard addition methods $(N=3)$. Upon inspection of Figure $5 F$, good linearity can be seen $\left(R^{2}\right.$ of 0.9979 and 0.9971 for AA and ACOP, respectively), and the concentration of the over-thecounter commercial tablets was experimentally calculated to have recovery values of 149.8 and $81.4 \%$ when compared to their theoretical concentration. It is important to note that when detecting simultaneously, our electrochemical sensor does not follow the same performance as when measuring independently AA and ACOP. This process is likely explained because of the electrochemical oxidation of AA being reported to be a surface-sensitive redox system, and although it is not affected by oxide species and does not require adsorption, AA oxidation is reported to have fouling effects on the surface of the electrode, which can explain the lower recovery results for ACOP. ${ }^{24,30}$ The all-in-one single print AM cell presented herein is designed as single use in terms of its electroanalysis application, and although this is common when working with ascorbic acid (due to sample degradation and electrode fouling), it is expected in the future the use of AM cells that can be regenerated after their use. Summarizing, herein, we report for the first time all-in-one single-print of a full electrochemical three-electrode cell and electrodes (including counter, reference, and working electrodes). It can be observed that our proposed single-print fully additive manufactured conductive carbon black/PLA is a successful electrochemical three-working electrode setup. We show that the electrochemical performance of the cell can be enhanced through standard chronoamperometric activation as seen throughout the literature, but crucially, this can be performed using the inbuilt counter and pseudo-reference electrodes, without the need for any external commercial electrodes. We have provided the heterogeneous electron transfer rate and explored the electroanalytical performance toward the important biomolecules ascorbic acid and acetaminophen, including their quantification in real samples from over-the-counter effervescent tablets, both individually and mixed, as an example of common electrochemical applications. Further exploration and improvements, such as antifouling properties, need exploration in the next steps of AM electrochemical platforms; however, this work shows the novelty, importance, and simplicity of prototyping electrochemical platforms by using AM, demonstrating that useful fully printed electrochemical setups can be achieved. We encourage other experimentalists to design their own all-in-one setups and sensor modifications to be applied to their target analytes by using this novel all-in-one easy to manufacture approach.

\section{CONCLUSIONS}

We have shown, for the first time, a fully functional singleprinted all-in-one additive manufactured electrochemical setup, including working, counter, and reference electrodes, that requires no post-assembly or external electrodes. We have also characterized the HET and electroanalytical properties of AM carbon black/PLA electrodes against the ideal outer-sphere redox probe RuHex, showing that excellent performance can be achieved directly off the print bed with no modification or assembly. We show that the electrode performance toward non-ideal probes can be improved by chemical activation via $\mathrm{NaOH}$ and chronoamperometry, which promotes PLA saponification exposing the material's active sites and electrochemical active area. Crucially, we show that this activation can be achieved through the use of the in-built pseudo-reference electrode, removing the need for any external/commercial electrodes. The sensor exhibited good repeatability and reproducibility and was successfully applied to the electrochemical determination of ascorbic acid (AA) and acetaminophen (ACOP) and their quantification in real over-the-counter effervescent tablets, achieving good results for their individual determination and their detection in mixed samples. We have shown how simple, electrochemical platforms can be produced in a single print, with all electrodes embedded into the watertight system. This work lays the foundation for how AM and electrochemistry can synergise and be applied to various research fields. 


\section{ASSOCIATED CONTENT}

\section{Supporting Information}

The Supporting Information is available free of charge at https://pubs.acs.org/doi/10.1021/acsmeasuresciau.1c00046.

(PDF)

STL files for different parts of the cell (ZIP)

\section{AUTHOR INFORMATION}

Corresponding Author

Craig E Banks - Faculty of Science and Engineering, Manchester Metropolitan University, Manchester M1 5GD, U.K.; (1) orcid.org/0000-0002-0756-9764; Phone: ++(0) 1612471196; Email: c.banks@mmu.ac.uk

\section{Authors}

Robert D. Crapnell - Faculty of Science and Engineering, Manchester Metropolitan University, Manchester M1 5GD, U.K.

Elena Bernalte - Faculty of Science and Engineering, Manchester Metropolitan University, Manchester M1 5GD, U.K.; $\odot$ orcid.org/0000-0002-0764-789X

Alejandro Garcia-Miranda Ferrari - Faculty of Science and Engineering, Manchester Metropolitan University, Manchester M1 SGD, U.K.; 우 orcid.org/0000-0003-1797-1519

Matthew J. Whittingham - Faculty of Science and Engineering, Manchester Metropolitan University, Manchester M1 5GD, U.K.

Rhys J. Williams - Faculty of Science and Engineering, Manchester Metropolitan University, Manchester M1 5GD, U.K.

Nicholas J. Hurst - Faculty of Science and Engineering, Manchester Metropolitan University, Manchester M1 5GD, U.K.

Complete contact information is available at:

https://pubs.acs.org/10.1021/acsmeasuresciau.1c00046

Notes

The authors declare no competing financial interest.

\section{ACKNOWLEDGMENTS}

The authors acknowledge the contributions of Hayley Andrews and Gary Miller for their work on the scanning electron microscopy and X-ray photoelectron spectroscopy, respectively. E.B. and A.G.-M.F. would like to acknowledge their respective Innovate UK funding for their Knowledge Transfer Partnerships (KTP Reference: 12021 and 11606, respectively).

\section{REFERENCES}

(1) Ryan, K. R.; Down, M. P.; Banks, C. E. Future of additive manufacturing: overview of $4 \mathrm{D}$ and $3 \mathrm{D}$ printed smart and advanced materials and their applications. Chem. Eng. J. 2021, 403, 126162.

(2) da Silveira, G. D.; Quero, R. F.; Bressan, L. P.; Bonacin, J. A.; de Jesus, D. P.; da Silva, J. A. F. Ready-to-use 3D-printed electrochemical cell for in situ voltammetry of immobilized microparticles and Raman spectroscopy. Anal. Chim. Acta 2021, 1141, 57-62.

(3) Dos Santos, M. F.; Katic, V.; Dos Santos, P. L.; Pires, B. M.; Formiga, A. L.; Bonacin, J. A. 3D-printed low-cost spectroelectrochemical cell for in situ Raman measurements. Anal. Chem. 2019, 91 (16), 10386-10389.

(4) Betlem, K.; Kaur, A.; Hudson, A. D.; Crapnell, R. D.; Hurst, G.; Singla, P.; Zubko, M.; Tedesco, S.; Banks, C. E.; Whitehead, K.; et al. Heat-Transfer Method: A Thermal Analysis Technique for the Real-
Time Monitoring of Staphylococcus aureus Growth in Buffered Solutions and Digestate Samples. ACS Applied Bio Materials 2019, 2 (9), 3790-3798.

(5) Crapnell, R. D.; Jesadabundit, W.; García-Miranda Ferrari, A.; Dempsey-Hibbert, N. C.; Peeters, M.; Tridente, A.; Chailapakul, O.; Banks, C. E. Toward the Rapid Diagnosis of Sepsis: Detecting Interleukin-6 in Blood Plasma Using Functionalized Screen-Printed Electrodes with a Thermal Detection Methodology. Anal. Chem. 2021, 93 (14), 5931-5938.

(6) Scremin, J.; Joviano dos Santos, I. V.; Hughes, J. P.; GarcíaMiranda Ferrari, A.; Valderrama, E.; Zheng, W.; Zhong, X.; Zhao, X.; Sartori, E. J. R.; Crapnell, R. D.; et al. Platinum nanoparticle decorated vertically aligned graphene screen-printed electrodes: electrochemical characterisation and exploration towards the hydrogen evolution reaction. Nanoscale 2020, 12 (35), 18214-18224.

(7) Whittingham, M. J.; Crapnell, R. D.; Rothwell, E. J.; Hurst, N. J.; Banks, C. E. Additive manufacturing for electrochemical labs: an overview and tutorial note on the production of cells, electrodes and accessories. Talanta Open 2021, 4, 100051.

(8) Cardoso, R. M.; Kalinke, C.; Rocha, R. G.; Dos Santos, P. L.; Rocha, D. P.; Oliveira, P. R.; Janegitz, B. C.; Bonacin, J. A.; Richter, E. M.; Munoz, R. A. Additive-manufactured (3D-printed) electrochemical sensors: A critical review. Anal. Chim. Acta 2020, 1118, 73-91.

(9) Redwood, B.; Schöffer, F.; Garret, B. The 3D printing handbook: technologies, design and applications; 3D Hubs, 2017.

(10) Gupta, V.; Alam, F.; Verma, P.; Kannan, A.; Kumar, S. Additive manufacturing enabled, microarchitected, hierarchically porous polylactic-acid/Lithium iron phosphate/carbon nanotube nanocomposite electrodes for high performance Li-Ion batteries. J. Power Sources 2021, 494, 229625.

(11) Hughes, J. P.; dos Santos, P. L.; Down, M. P.; Foster, C. W.; Bonacin, J. A.; Keefe, E. M.; Rowley-Neale, S. J.; Banks, C. E. Single step additive manufacturing (3D printing) of electrocatalytic anodes and cathodes for efficient water splitting. Sustainable Energy \& Fuels 2020, 4 (1), 302-311.

(12) Foster, C. W.; Elbardisy, H. M.; Down, M. P.; Keefe, E. M.; Smith, G. C.; Banks, C. E. Additively manufactured graphitic electrochemical sensing platforms. Chem. Eng. J. 2020, 381, 122343.

(13) Rocha, D. P.; Ataide, V. N.; de Siervo, A.; Gonçalves, J. M.; Muñoz, R. A.; Paixão, T. R.; Angnes, L. Reagentless and sub-minute laser-scribing treatment to produce enhanced disposable electrochemical sensors via additive manufacture. Chem. Eng. J. 2021, 425, 130594.

(14) Redondo, E.; Muñoz, J.; Pumera, M. Green activation using reducing agents of carbon-based 3D printed electrodes: Turning good electrodes to great. Carbon 2021, 175, 413-419.

(15) Giorgini Escobar, J.; Vaněčková, E.; Nováková Lachmanová, Š. N.; Vivaldi, F.; Heyda, J.; Kubišta, J.; Shestivska, V.; Španěl, P.; Schwarzová-Pecková, K.; Rathouský, J.; et al. The development of a fully integrated $3 \mathrm{D}$ printed electrochemical platform and its application to investigate the chemical reaction between carbon dioxide and hydrazine. Electrochimica acta 2020, 360, 136984.

(16) Symes, M. D.; Kitson, P. J.; Yan, J.; Richmond, C. J.; Cooper, G. J.; Bowman, R. W.; Vilbrandt, T.; Cronin, L. Integrated 3D-printed reactionware for chemical synthesis and analysis. Nat. Chem. 2012, 4 (5), 349-354.

(17) Ambrosi, A.; Pumera, M. Self-contained polymer/metal 3D printed electrochemical platform for tailored water splitting. $A d v$. Funct. Mater. 2018, 28 (27), 1700655.

(18) Lee, C. Y.; Taylor, A. C.; Beirne, S.; Wallace, G. G. A 3Dprinted electrochemical water splitting cell. Advanced Materials Technologies 2019, 4 (10), 1900433.

(19) Elbardisy, H. M.; Richter, E. M.; Crapnell, R. D.; Down, M. P.; Gough, P. G.; Belal, T. S.; Talaat, W.; Daabees, H. G.; Banks, C. E. Versatile additively manufactured (3D printed) wall-jet flow cell for high performance liquid chromatography-amperometric analysis: application to the detection and quantification of new psychoactive substances (NBOMes). Anal. Methods 2020, 12 (16), 2152-2165. 
(20) Ramiah Rajasekaran, P.; Chapin, A. A.; Quan, D. N.; Herberholz, J.; Bentley, W. E.; Ghodssi, R. 3D-Printed electrochemical sensor-integrated transwell systems. Microsystems \& Nanoengineering 2020, 6 (1), 100.

(21) Richter, E. M.; Rocha, D. P.; Cardoso, R. M.; Keefe, E. M.; Foster, C. W.; Munoz, R. A.; Banks, C. E. Complete additively manufactured (3D-printed) electrochemical sensing platform. Anal. Chem. 2019, 91 (20), 12844-12851.

(22) Nicholson, R. S. Theory and application of cyclic voltammetry for measurement of electrode reaction kinetics. Anal. Chem. 1965, 37 (11), 1351-1355.

(23) Bard, A. J.; Faulkner, L. R. Electrochemical methods: Fundamentals and Applications, 2nd ed.; Wiley, 2000.

(24) García-Miranda Ferrari, A.; Foster, C. W.; Kelly, P. J.; Brownson, D. A.; Banks, C. E. Determination of the electrochemical area of screen-printed electrochemical sensing platforms. Biosensors 2018, 8 (2), 53.

(25) Randles, J. E. A cathode ray polarograph. Part II.-The currentvoltage curves. Trans. Faraday Soc. 1948, 44, 327-338.

(26) Ševčík, A. Oscillographic polarography with periodical triangular voltage. Collect. Czech. Chem. Commun. 1948, 13, 349-377.

(27) Ferrari, A. C.; Basko, D. M. Raman spectroscopy as a versatile tool for studying the properties of graphene. Nat. Nanotechnol. 2013, 8 (4), 235-246.

(28) Ferrari, A. C.; Meyer, J. C.; Scardaci, V.; Casiraghi, C.; Lazzeri, M.; Mauri, F.; Piscanec, S.; Jiang, D.; Novoselov, K. S.; Roth, S.; et al. Raman spectrum of graphene and graphene layers. Physical review letters 2006, 97 (18), 187401.

(29) Graf, D.; Molitor, F.; Ensslin, K.; Stampfer, C.; Jungen, A.; Hierold, C.; Wirtz, L. Raman imaging of graphene. Solid State Commun. 2007, 143 (1-2), 44-46.

(30) McCreery, R. L. Advanced carbon electrode materials for molecular electrochemistry. Chem. Rev. 2008, 108 (7), 2646-2687. 